\title{
Review on Gene regulation: DNA-protein and protein- protein interactions and their regulatory elements
}

\author{
Safir Ullah Khan 1, ${ }^{*}$, Munir Ullah Khan² \\ ${ }^{1}$ Hefei National Laboratory for Physical Sciences at the Microscale, School of Life Sciences, University \\ ofScience and Technology of China, Hefei 230027, China \\ ${ }^{2}$ MOE Key Laboratory of Macromolecular Synthesis and Functionalization, Department of Polymer Science \\ and Engineering, Zhejiang University, Hangzhou 310027, China
}

Received: 01.10.2021 • Accepted: 20.10.2021 • Published: 23.10.2021 • Final Version: 29.10.2021

\begin{abstract}
The non-coding elements that control transcription are found in the chromatin structure of organisms. Recent findings identify the non-coding regulatory elements (e.g., enhancers, silencers, promoters) that control transcription and examine their respective protein interactions. The multiple topological environment limitations, including interactions of promoter-enhancer and specific enhancer-bound proteins with variable promoter compatibility, begin to shape a picture. These transcription factors and co-factors contribute to various expressions based on which enhancers and promoters are found inside sequences. A novel trait of transcription factors and cofactors establishes nuclear microenvironments or membranes compartments with phase-separated liquid characteristics. These settings are capable of enriching some proteins and tiny molecules at the expense of others. To better understand gene regulation in the nucleus, more study is needed on transcriptional regulators and their interactions.
\end{abstract}

Keywords: transcription, protein interactions, transcription factors, transcriptional regulators.

\section{Introduction}

Specific gene expression systems need the establishment and maintenance in multicellular organisms. Transcription regulates gene expression by converting DNA to RNAAt promoter regions, RNA polymerase II (Pol II) initiates the first stage of RNA synthesis, because Pol II is only present in promoter regions before transcription starts. When the pre-initiation complex (PIC) is present, only low amounts of transcription are achievable. To greatly increase the of effective transcriptional initiation, enhancer regions of non-coding regulatory are also required. An enhancer adds proteins such as transcription factors (T.F.s) and co-factors to an existing transcription unit, increasing the frequency of transcription from the unit's promoter. To understand how gene expression programs are regulated, it is critical to investigate the molecular mechanisms by which these factors interact to activate transcription.

This section will explore new ideas about examining the transcription process and recent findings that help explain how regulatory specificity is achieved. Our final focus is on the biophysical ability of transcription factors and co-factors to establish liquid-liquid phase separation microenvironments to assist protein-protein interaction with the regulatory element to which it connects.

\footnotetext{
* Corresponding Author: safir@ mail.ustc.edu.cn
} 


\subsection{Dynamics of transcriptional bursting}

Despite the fact that transcription levels vary considerably between genes, the mechanism by which such different transcription rates are regulated is still a mystery to researchers. Most transcription is a series of bursts separated by periods of apparent inactivity, using two model methods: burst size (the RNA generated by each burst) and also burst frequency (In a certain time frame, how many bursts occur is recorded)[1,2]. Thus, it is possible to vary the transcription parameters by adjusting the length, frequency, or both of the bursts.

Researchers recently gained better insights into nascent transcripts and can assist in identifying the bursting dynamics of single genes with the recent advancements in live-cell imaging. For example, the estrogen-responsive TFF1 gene[3], with short and extended refractory periods of several hours to many days, can perhaps explain the erratic nature of populations of individual cells[4]. Additionally, imaging is applied to measure the mechanisms that may influence or govern bursting dynamics. In particular, the T.F.s' residence durations and the bonding percentages were linked with bursting Through the Gal4 transcription factors and the glucocorticoid receptors [5, 6]. This discovery was somewhat surprising as the modification of bursting parameters such as frequency and burst size did not appear to depend on Pol II recruitment since cellular differentiation or the inhibition of BET protein altered the frequency of burst size; However, the recruiting rates for Pol II did not effect[7]. Thus, the data reveal that some promoters appear to use a separate rate-limiting phase different from Pol II recruiting to set off transcription bursts.

A significant improvement has been made in the parallel analysis of thousands of expressed genes using allele resolved single-cell RNA sequencing. [8], which is likely to result in highly accurate estimates of transcriptional burst characteristics. By studying gene expression at the genome level, this analysis revealed that the activity of an enhancer was found to affect burst frequency. However, the size of a promoter affected TATA box promoters and burst size. Scientist believes that it will shed new light on the regulation of bursting.

\subsection{Gene expression and genome topology}

Gene expression is best described as gene and regulatory components being bound into localized regions known as topologically associated domains (TADs). Intra-TAD chromatin interaction frequencies are high, but inter-TAD chromatin interaction frequencies are low. TADs may act as functional units to guide transcriptional regulation because they can allow intra-TAD regulatory communication by interacting with surrounding TAD regulatory components. TAD-functioning examples come from structural polymorphism analysis linked to different medical problems [9-13]. By examining a branchiooculofacial syndrome model, scientists determined that an inversion disrupted a TAD comprising the TFAP2A gene. As a result, TFAP2A production was reduced. Most likely, the promoter of TFAP2A was detached from its corresponding enhancers[14]. An unusual inverse relationship occurred between the Epha4 locus and gene expression; when new connections developed in the Epha4 locus, The new interactions with enhancers from a neighboring gene cluster resulted in aberrant gene expression [15]. Overall, these occurrences of disease-associated structural variations suggest that topological boundaries play a role in ensuring the robustness of gene expression and preventing abnormal enhancer-promoter interactions.

The several TAD disruptions discussed here prove that TADs are required for gene expression maintenance and can shed insight on several previously unknown disease characteristics. Furthermore, most other studies indicated that altering TAD structures had no substantial effect on gene expression; TAD fusion was generated by changes in the CTCF sites adjacent TADs within 
Sox9-Kcnj2 locus, although gene expression was low or non-existent as a result of these changes. [16]. Hox-D gene expression is similar after removing boundaries in the Hox-D locus[17], and after many years of structural rearrangements, gene expression on Drosophila balancer chromosomes is also intact[18]. However, in the vicinity of breakpoints, Hox-D gene expression has changed in its levels and patterns of expression [19].

An identical pattern of gene expression change was also observed when TADs were rapidly depleted, removing the possibility of compensating mechanisms being used. During acute depletion of cohesin in mESCs, the formation of TADs was disrupted, leading to the elimination of domain [20]. However, relatively minor alterations were detected in gene expression, confined to genes located near super-enhancers. While the decrease of the protein CTCF with only a minor impact on gene expression led to the complete elimination of TADs, it also eliminated CTCF, whose abundance had previously established boundaries for genes[21, 22].

While the exact mechanisms underlying these disparate outcomes are unknown and may include the development of new links rather than the severance of existing ones [16]. Gene expression looks remarkably stable; however, it has not yet been demonstrated that this is consistently the case. In addition to these results demonstrate that, in most cases, a single TAD does not control the Prevalence of enhancer-promoter contacts across multiple genes[23]. This research supports the conclusion that new mechanisms must be developed to account for how individual TADs govern the rate of enhancer-promoter interactions in cells.

inversion leading to enhancer-promoter rewiring
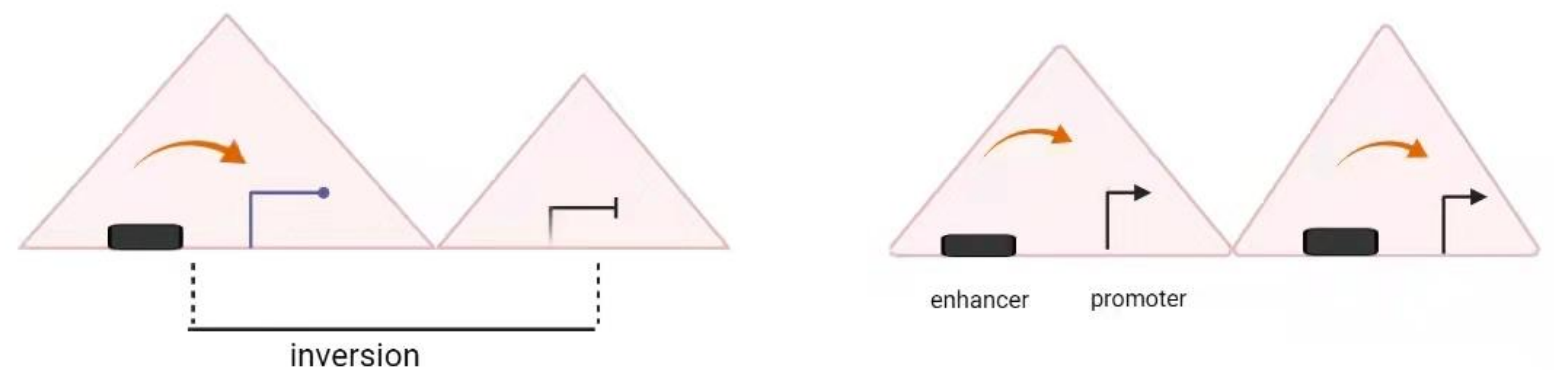

enhancer promoter

inversion
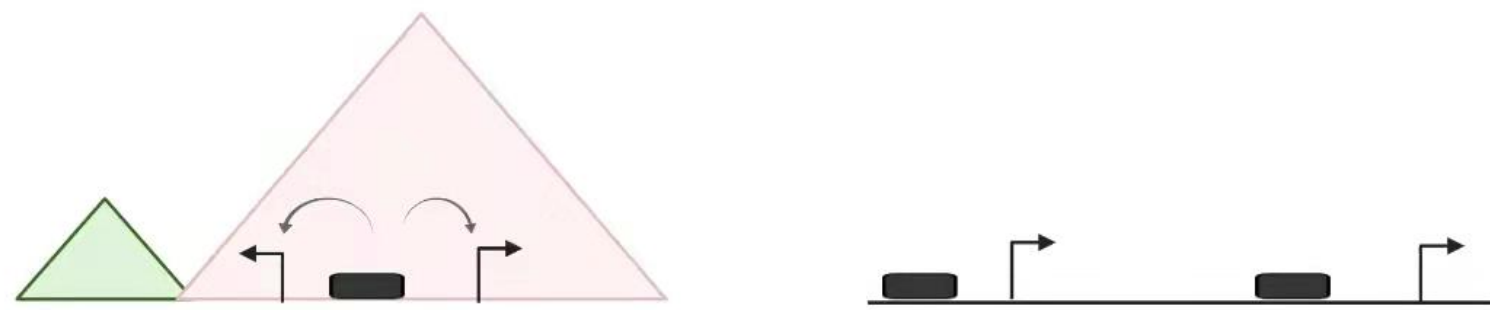

Figure 1.TADs limit the interactions between enhancers and promoters. By rewiring promoter-enhancer connections, these alterations to the structure, like inversions, may affect the regulation of gene expression. On the other hand, a full breakdown of the TAD structure has no tangible effect on gene expression. 


\subsection{Co-factors involved in transcription}

Although this happens in many disease-associated examples mentioned, abnormal connections between Enhancer and promoter resulted in the gene expression of just certain in certain situations. However, this may mean there is an intrinsic trait present in enhancers and promoters in not all genes. The similarity between Drosophila regulatory elements has indeed been shown in the experiment where a promoter with a set of genome-wide enhancers is paired with a promoter belonging to a different species[24, 25]. In humans and Drosophila, transcriptiona coactivators reconcile enhancerpromoter communication and transcriptional start. When coactivators like mediators or MLL3/4 were driven to recruit a complicated library of promoters, they preferentially activated distinct promoters. Because of these preferences for co-factors and their inability to universally fit all promoters[26], It is reasonable to believe that distinct co-factors use various methods to control a variety of different gene expression and these pathway are not interchangeable between co-factors. Promoters that react uniquely to the list of previously discussed co-factors have sequence variations, initiator motifs, and TATA-box , for example, be effective against $\mathrm{CpG}$ dinucleotides. There are multiple regulatory and rate-limiting factors associated with variations of these features. Thus these characteristics will likewise vary in those stages. [27], They may interact with a variety of proteins and/or have different PICs. The application of regulatory compatibilities to various promoter types and how promoter-enhancers work are addressed, and co-factors eventually influence transcriptional bursts.

Many transcription factors (promoters and co-factors) can share regulatory compatibilities, implying that transcriptional regulation could differ according to different factors. Several examples of differential co-factor requirements have been seen in the last years. For example, in olfactory epithelial cells, the co-factor Ldb1 and transcriptional regulators Lhx2 are required for olfactory receptor gene transcription. [28]. Similarly, the coactivator-3 (SRC-3) of an estrogen receptor is used particularly to activate the GREB1 gene[29]. Without the activities of the co-factors, transcription would be impaired, or the spatial relationships between the promoters and their analogous enhancers would be disrupted. Even at the levels of each subunit of the mediator, gene-specific co-factor requirements are seen. Studies conducted in the last decade indicated that depletion of MED14, a structural core mediator component, caused a global disruption of gene expression without changing the topological structure of the genome. [30].

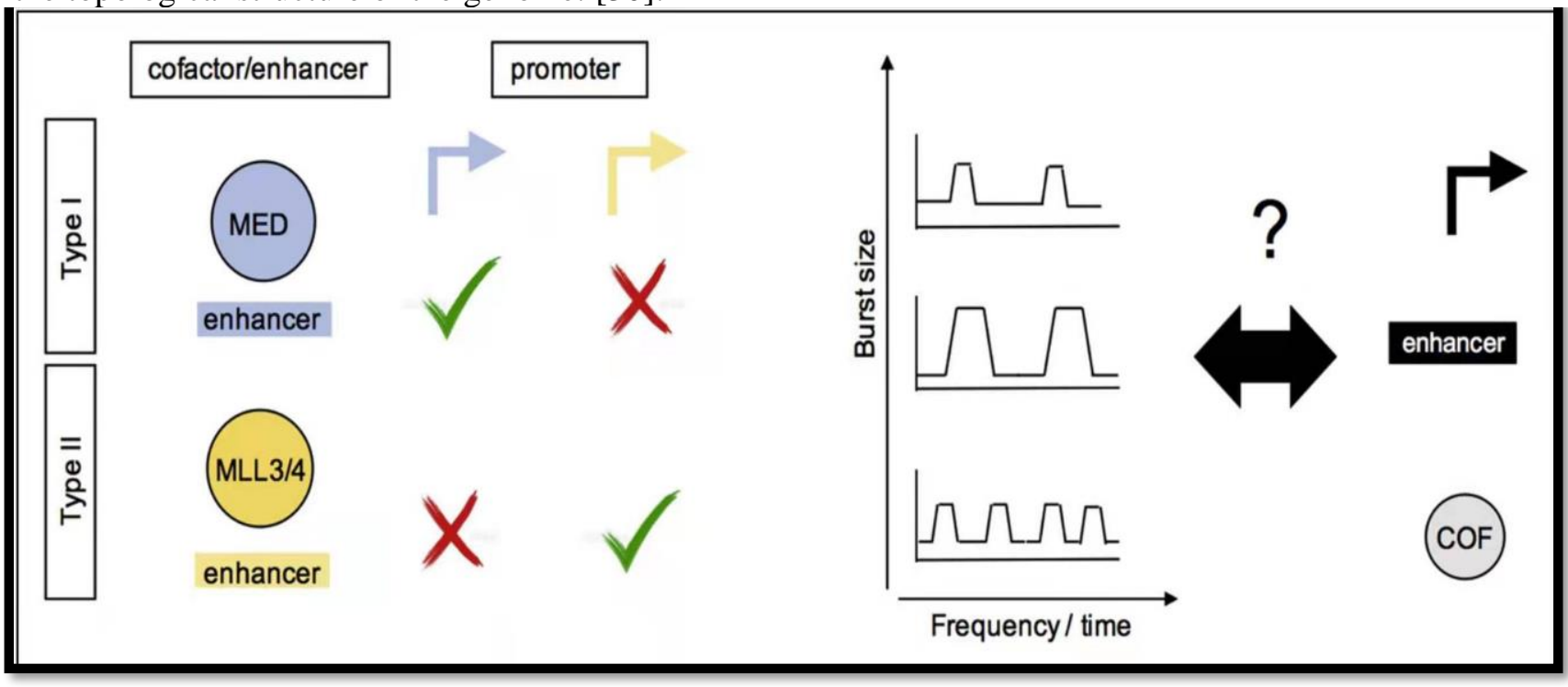


Figure 2.Different types of promoters and enhancers communicate by various co-factors. TATAbox and CpG island promoter types have been demonstrated to be selectively activated by stimulating co-factors such as mediator and MLL3/4, responding differentially to specific Enhancer. Ongoing research is focused on the relationships between enhancers, promoters, and cofactors (grey circle) and the dynamics of transcriptional bursts.

These alterations in gene expression and cell viability were caused by decreased mediator units (particularly in the tail loop).According to these investigations, mediator subcomponents may exist and may be utilized differently[30]. The current study found that co-factors are unnecessary for all enhancers and promoters to work, and transcription may activate processes using co-factors that are unique to those particular mechanisms.

\subsection{Transcription factors.}

One of the most important links between regulatory DNA elements and co-factors is transcription factors; also, because they influence gene regulation and co-factor specificity, they can manipulate the expression of genes. The structure of T.F.s reflects this dual function, which consists of a DNA binding domain (DBD) and a transactivation domain (TAD) to facilitate in activating the transcription factor via co-factor binding and recruitment.

T.F.s must have very specific criteria for identifying their specific binding sites, as regulatory elements are frequently entombed in chromatin. The capacity of 220 transcription factors to attach chromatinized DNA was profiled[31]. It was discovered that the vast majority of transcription factors are unable to reach chromatinized DNA. Several T.F.s, including those in the EN1 and SOX families[32], bind tightly to nucleosomal DNA and are classified as pioneer factors with others like FoxA in humans, Grainyhead, and Zelda in Drosophila[33, 34]. Structural studies reveal that pioneer T.F.s can engage chromatinized DNA and then serve as a platform for binding additional T.F.s. The linkage of pioneering T.F.s such as Sox 2 and Sox 11 with nucleosomal DNA leads to distortions in DNA. To help with the binding of the transcription factors, the nucleosome is destabilized, and a portion of the motif binding sites are subjected to allow binding. [35-38]. In addition, the repositioning of pioneering T.F.s by the $\mathrm{N}$-terminals of histone 4 is binding, which could be incompatible with greater nuclear stacking, thus opening up local chromatization.

The T.F.s' transcription activation domains (tADs) are essential for transcriptional activation because of their interactions with co-factors. In recent years, Not only have these domains been mapped and dissected using high-throughput methods in recent years but, a role for tADs in influencing T.F. binding in vivo appears to exist irrespective of the DNA-binding domains of the transcription factors. [39]. The latter illustration demonstrated that Msn2 deletion did not impact its genomic synchronization at most binding site. It is questionable whether the $\mathrm{tAD}$ recognizes specific targets via DNA association sampling or by interacting with other T.F.s bound to Msn2-target promoters, as suggested for highly occupied target (HOT) regions[40].

The combinatorial function of transcription factors (T.F.s) is a fundamental part of developmental gene regulation[41]. The general scientific consensus has long been that T.F.s bind cooperatively, like single nucleotide polymorphisms (SNPs) or mutations in a T.F. binding site might alter the affinity of another T.F. [42-44]. For this reason, we completely unaware of the dynamics of co-binding and how many transcription factors are co-bound at the same moment. Multiple DNA footprinting technologies now permit the simultaneous detection of T.F. attachment and DNA accessibility of adjacent enhancers [45-47]. Remarkably, adjacent T.F. binding sites and enhancers tend to coordinate with each other. Apart from the cooperative attachment of co-expressed T.F.s, To 
enhance the establishment of asymmetric neuron pairs in C. elegans, even T.Fs that never co-express can induce transcription in a creative manner via a temporal integration mechanism[48].

\subsection{Selective cofactor-TF interactions by liquid-liquid phase separation}

We have mostly been under the influence of a sequential model that holds that unique transcription factors bind to specific DNA sequences, followed by specific protein-protein interaction that bind co-factors homogeneously distributed inside the nucleus. This model has been improved during the last years by a developing biophysical property that many regulators display the capability of becoming liquid-liquid phase separators (LLPs)[49].Changes in histone modifications and readers of those modifications may also induce multivalent relationships, such as the one found between histone and their reader-domains[50]; generate new microenvironments that focus on specific factors. Indeed HP1 LLPs and H3K9me3 not only induce heterochromatin formation but are also important in causing the increased compactness of this kind of chromatin, as well as inhibiting transcription factor access to the chromatin domains[51-53].

Interestingly, not all HP1-distinct heterochromatin appears to follow the LLPS form, suggesting that heterochromatin contraction may occur independently of HP1[54]. More broadly, the LLPS model can be used to segment engaged and repressed genomic regions: Clusters of H3K27-acetylated chromatin domains are found in C.elegans. They are linked to p300/CBP-1, an acetyltransferase, and ATF-8, a transcriptional activator[55].Active domains of DNA methylation and CEC-4 are structurally different from repressed H3K9-methylated areas. The loss of CEC-4 or Mrg1 resulted in the extension of p300/CBP-1 in heterochromatin.

Additionally, maintaining active and repressed domains is strongly dependent on a proper balance among repressors and activators. Recently, MeCP2 is enriched at HP1 indicated LLPS heterochromatin areas[56]. Patients with Rett syndrome who possess mutations in the MeCP2 gene have impaired ability to phase separate MeCP2, indicating that LPS may play a role in the disease process[57]. In addition, it is not always the case that transcription is detected at transcription sites, and it will be significant to comprehensively compare all the transcription sites found with those in LLPS condensates [58-62].

The existence of acetylated lysines on Histone 3 can make an important contribution to chromatin LLPS capability. Chromatin droplets dissolve due to the reduction in net positive charge caused by acetylating nucleosomes on lysine residues [63]. On the other hand, acylated chromatin can phase segregate into droplets in the existence of the activator Brd4, which excludes parts of the chromatin that Brd4 does not attach in vitro. Phase separation and segmentation of distinct phases are likely enhanced by chemical perturbations on chromatin, including the ubiquitination, methylation, and phosphorylation of chromatin as well as the Partitioning of different phases to allow precise proteinprotein interaction[64].

There are many transcriptional proteins that could use LLPS including Pol II[65], mediators (particularly Med1)[66], and transcription factors (T.F.s)[67]. The intrinsically disordered regions (IDRs) that may play a significant role in LLPS are also predicted to be present in low-complexity domains. Although mutations that alter the length of individual I.D.s are harmless for specific T.F.s, they can change the activation functions and tendency to phase separate for particular transcription factors [68, 69]. After forming, these condensates have the ability to bind proteins and tiny molecules: It has been demonstrated that mediator condensates can accumulate signaling molecules like as SMAD3 and STAT3 [70], The DNA binding co-factor TEAD4 as well as other co-factors including such Brd4, p300, and Cdk9 were enriched in droplets generated by the TF TAZ [71]. There 
are many other molecules aside from proteins that also contribute to the concentration of certain activators. Examples include small molecules called LLPs, such as Med1 LLPs droplets, which concentrate on therapeutic agents such as cisplatin[72].

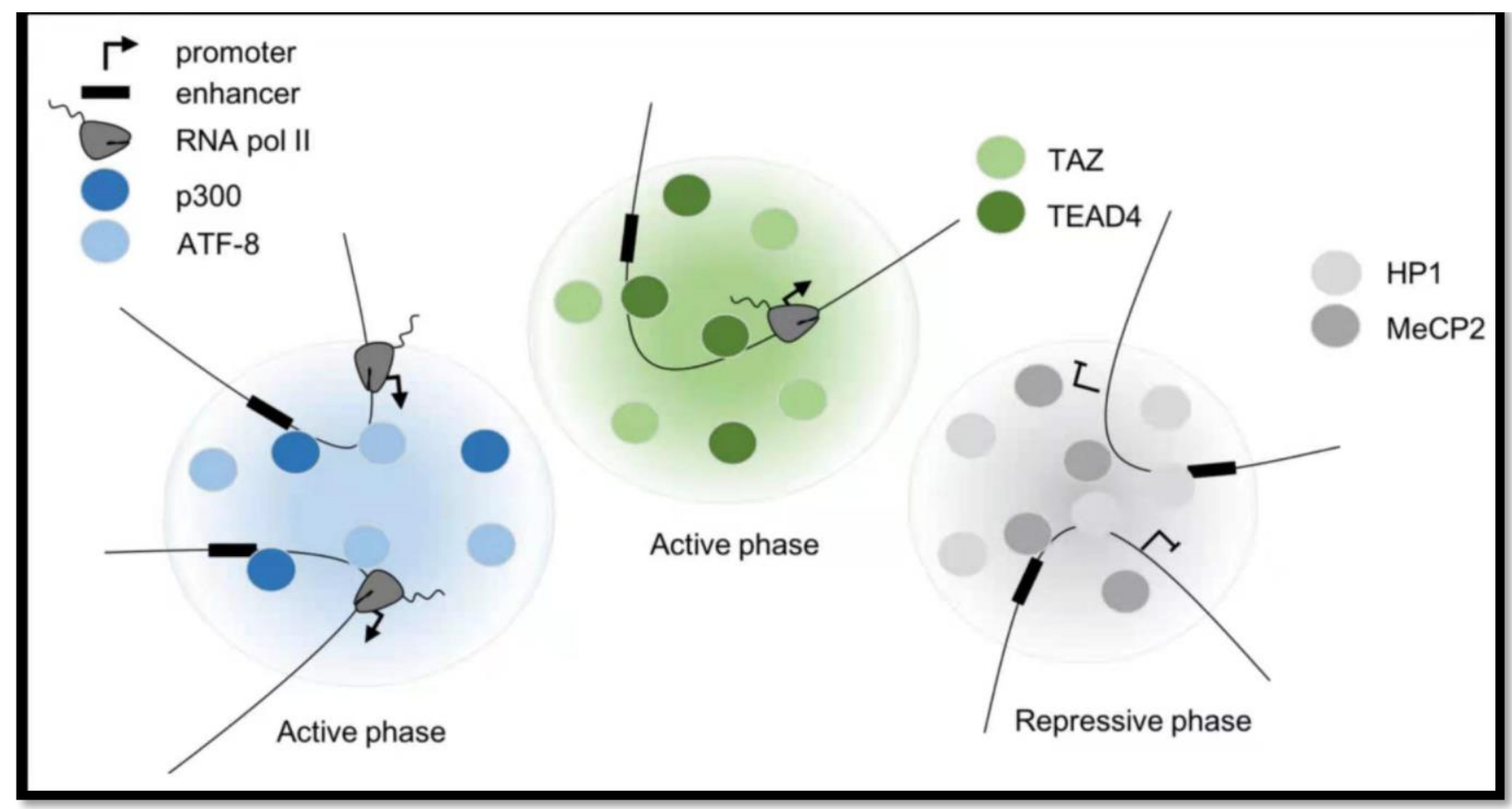

Figure 3. A variety of transcription factors and co-factors take part in various protein-protein interaction in LLPS droplets, which can result in the formation of transcriptionally repressed (grey) or transcriptionally active (green) phases (blue and green).

Knowing the several different types of nuclear environments and the chemical laws that regulate their development would be quite fascinating (Figure 3). For example, we can imagine that distinct nuclear functions, such as transcription repression and activation, may generate their own nuclear microenvironment. This could lead to two separate ways of activation by developing and housekeeping transcriptional processes [73]. Respectively, mitosis, DNA damage, or other factors may be involved. Though heterochromatin phase transitions create large domains, activation appears to produce smaller-sized microenvironments or hubs. Consequently, Researchers are trying to figure out how many proteins are involved and how they interact with each other including an electron microscope or high-resolution optical microscopy, would be required [74-76].

\section{Conclusion}

Gene regulation is a complex procedure that involves numerous protein components that influence non-coding regulatory elements. This study finds that understanding the relationship between genomic architecture and Enhancer and regulatory domains has progressed greatly in the last several years. Further progress will be fascinating to grasp how different promoter types convert activation to transcription function that how these indications affect burst sizes or frequencies, or both, to increase transcriptional activity.

We believe these LLPS research results will also reveal novel insights into the molecular principles that regulate the division of the nucleus into smaller microdomains, which allow various proteins 
and their abilities to be enriched while restricting other proteins and their functions. New, highresolution imaging technologies will allow for more comprehensive investigations of these kinds of LLPS at the site of active or repressed gene loci.

\section{Acknowledgements}

We thank the members of the Journal of Chemistry and Nutritional Biochemistry for helpful comments and discussion.

\section{Author contribution}

Safir Ullah Khan devised and designed the study. Munir Ullah Khan performed survey and analysis data. Authors read and approved the manuscript.

\section{Declarations}

Conflict of interestthe authors declare no potential conflicts of interest.

Ethical approval This Article does not contain any studies with human participants or animals performed by the author.

\section{References}

[1] Abdulhay, Nour J, McNally, Colin P, Hsieh, Laura J, Kasinathan, Sivakanthan, Keith, Aidan, Estes, Laurel S, . . . Narlikar, Geeta J. (2020). Massively multiplex single-molecule oligonucleosome footprinting. Elife, 9, e59404.

[2] Allen, Benjamin L, \& Taatjes, Dylan J. (2015). The Mediator complex: a central integrator of transcription. Nature reviews Molecular cell biology, 16(3), 155-166.

[3] Arnold, Cosmas D, Zabidi, Muhammad A, Pagani, Michaela, Rath, Martina, Schernhuber, Katharina, Kazmar, Tomáš, \& Stark, Alexander. (2017). Genome-wide assessment of sequenceintrinsic enhancer responsiveness at single-base-pair resolution. Nature biotechnology, 35(2), 136-144.

[4] Banani, Salman F, Lee, Hyun O, Hyman, Anthony A, \& Rosen, Michael K. (2017). Biomolecular condensates: organizers of cellular biochemistry. Nature reviews Molecular cell biology, 18(5), 285-298.

[5] Bardet, Anaïs F, He, Qiye, Zeitlinger, Julia, \& Stark, Alexander. (2012). A computational pipeline for comparative ChIP-seq analyses. Nature protocols, 7(1), 45-61.

[6] Bartman, Caroline R, Hamagami, Nicole, Keller, Cheryl A, Giardine, Belinda, Hardison, Ross C, Blobel, Gerd A, \& Raj, Arjun. (2019). Transcriptional burst initiation and polymerase pause release are key control points of transcriptional regulation. Molecular cell, 73(3), 519-532. e514.

[7] Basu, Shaon, Mackowiak, Sebastian D, Niskanen, Henri, Knezevic, Dora, Asimi, Vahid, Grosswendt, Stefanie, Ewers, Helge. (2020). Unblending of transcriptional condensates in human repeat expansion disease. Cell, 181(5), 1062-1079. e1030.

[8] Boehning, Marc, Dugast-Darzacq, Claire, Rankovic, Marija, Hansen, Anders S, Yu, Taekyung, Marie-Nelly, Herve, Cramer, Patrick. (2018). RNA polymerase II clustering through carboxyterminal domain phase separation. Nature structural \& molecular biology, 25(9), 833-840.

[9] Boija, Ann, Klein, Isaac A, Sabari, Benjamin R, Dall'Agnese, Alessandra, Coffey, Eliot L, Zamudio, Alicia V, Hannett, Nancy M. (2018). Transcription factors activate genes through the phase-separation capacity of their activation domains. Cell, 175(7), 1842-1855. e1816. 
[10] Brodsky, Sagie, Jana, Tamar, Mittelman, Karin, Chapal, Michal, Kumar, Divya Krishna, Carmi, Miri, \& Barkai, Naama. (2020). intrinsically disordered regions direct transcription factor in vivo binding specificity. Molecular cell, 79(3), 459-471. e454.

[11] Cabianca, Daphne S, Muñoz-Jiménez, Celia, Kalck, Véronique, Gaidatzis, Dimos, Padeken, Jan, Seeber, Andrew, Gasser, Susan M. (2019). Active chromatin marks drive spatial sequestration of heterochromatin in C. elegans nuclei. Nature, 569(7758), 734-739.

[12] Charest, Julien, Daniele, Thomas, Wang, Jingkui, Bykov, Aleksandr, Mandlbauer, Ariane, Asparuhova, Mila, Cochella, Luisa. (2020). Combinatorial action of temporally segregated transcription factors. Developmental cell, 55(4), 483-499. e487.

[13] Chong, Shasha, Dugast-Darzacq, Claire, Liu, Zhe, Dong, Peng, Dailey, Gina M, Cattoglio, Claudia, Darzacq, Xavier. (2018). Imaging dynamic and selective low-complexity domain interactions that control gene transcription. Science, 361(6400).

[14] Chubb, Jonathan R, Trcek, Tatjana, Shenoy, Shailesh M, \& Singer, Robert H. (2006). Transcriptional pulsing of a developmental gene. Current biology, 16(10), 1018-1025.

[15] Clarke, Jane, \& Pappu, Rohit V. (2017). Editorial overview: Protein folding and binding, complexity comes of age. Current opinion in structural biology, 42, v-vii.

[16] De Laat, Wouter, \& Duboule, Denis. (2013). Topology of mammalian developmental enhancers and their regulatory landscapes. Nature, 502(7472), 499-506.

[17] Deplancke, Bart, Alpern, Daniel, \& Gardeux, Vincent. (2016). The genetics of transcription factor DNA binding variation. Cell, 166(3), 538-554.

[18] Despang, Alexandra, Schöpflin, Robert, Franke, Martin, Ali, Salaheddine, Jerković, Ivana, Paliou, Christina, Vingron, Martin. (2019). Functional dissection of the Sox9-Kcnj2 locus identifies nonessential and instructive roles of TAD architecture. Nature genetics, 51(8), 1263 1271.

[19] Dixon, Jesse R, Selvaraj, Siddarth, Yue, Feng, Kim, Audrey, Li, Yan, Shen, Yin, .. . Ren, Bing. (2012). Topological domains in mammalian genomes identified by analysis of chromatin interactions. Nature, 485(7398), 376-380.

[20] Dodonova, Svetlana O, Zhu, Fangjie, Dienemann, Christian, Taipale, Jussi, \& Cramer, Patrick. (2020). Nucleosome-bound SOX2 and SOX11 structures elucidate pioneer factor function. Nature, 580(7805), 669-672.

[21] Donovan, Benjamin T, Huynh, Anh, Ball, David A, Patel, Heta P, Poirier, Michael G, Larson, Daniel R, . . . Lenstra, Tineke L. (2019). Live-cell imaging reveals the interplay between transcription factors, nucleosomes, and bursting. The EMBO journal, 38(12), e100809.

[22] Erdel, Fabian, Rademacher, Anne, Vlijm, Rifka, Tünnermann, Jana, Frank, Lukas, Weinmann, Robin, Bauer, Caroline. (2020). Mouse heterochromatin adopts digital compaction states without showing hallmarks of HP1-driven liquid-liquid phase separation. Molecular cell, 78(2), 236-249. e237.

[23] Gallego, Laura D, Schneider, Maren, Mittal, Chitvan, Romanauska, Anete, Carrillo, Ricardo M Gudino, Schubert, Tobias, Köhler, Alwin. (2020). Phase separation directs ubiquitination of gene-body nucleosomes. Nature, 579(7800), 592-597.

[24] Garcia, Meilin Fernandez, Moore, Cedric D, Schulz, Katharine N, Alberto, Oscar, Donague, Greg, Harrison, Melissa M, . . Zaret, Kenneth S. (2019). Structural features of transcription factors associating with nucleosome binding. Molecular cell, 75(5), 921-932. e926.

[25] Gemayel, Rita, Chavali, Sreenivas, Pougach, Ksenia, Legendre, Matthieu, Zhu, Bo, Boeynaems, Steven, Schymkowitz, Joost. (2015). Variable glutamine-rich repeats modulate transcription factor activity. Molecular cell, 59(4), 615-627.

[26] Ghavi-Helm, Yad, Jankowski, Aleksander, Meiers, Sascha, Viales, Rebecca R, Korbel, Jan O, \& Furlong, Eileen EM. (2019). highly rearranged chromosomes reveal uncoupling between genome topology and gene expression. Nature genetics, 51(8), 1272-1282.

[27] Gibcus, Johan H, Samejima, Kumiko, Goloborodko, Anton, Samejima, Itaru, Naumova, Natalia, Nuebler, Johannes, Earnshaw, William C. (2018). A pathway for mitotic chromosome formation. Science, 359(6376). 
[28] Gibson, Bryan A, Doolittle, Lynda K, Schneider, Maximillian WG, Jensen, Liv E, Gamarra, Nathan, Henry, Lisa, . . . Rosen, Michael K. (2019). Organization of chromatin by intrinsic and regulated phase separation. Cell, 179(2), 470-484. e421.

[29] Guerrero-Martínez, Jose A, Ceballos-Chávez, María, Koehler, Florian, Peiró, Sandra, \& Reyes, Jose C. (2020). TGF $\beta$ promotes widespread enhancer chromatin opening and operates on genomic regulatory domains. Nature communications, 11(1), 1-20.

[30] Haberle, Vanja, Arnold, Cosmas D, Pagani, Michaela, Rath, Martina, Schernhuber, Katharina, \& Stark, Alexander. (2019). Transcriptional cofactors display specificity for distinct types of core promoters. Nature, 570(7759), 122-126.

[31] Haberle, Vanja, \& Stark, Alexander. (2018). Eukaryotic core promoters and the functional basis of transcription initiation. Nature reviews Molecular cell biology, 19(10), 621-637.

[32] Heinz, Sven, Benner, Christopher, Spann, Nathanael, Bertolino, Eric, Lin, Yin C, Laslo, Peter, Glass, Christopher K. (2010). Simple combinations of lineage-determining transcription factors prime cis-regulatory elements required for macrophage and B cell identities. Molecular cell, 38(4), 576-589.

[33] Iwafuchi-Doi, Makiko, Donahue, Greg, Kakumanu, Akshay, Watts, Jason A, Mahony, Shaun, Pugh, B Franklin, Zaret, Kenneth S. (2016). The pioneer transcription factor FoxA maintains an accessible nucleosome configuration at enhancers for tissue-specific gene activation. Molecular cell, 62(1), 79-91.

[34] Jacobs, Jelle, Atkins, Mardelle, Davie, Kristofer, Imrichova, Hana, Romanelli, Lucia, Christiaens, Valerie, Taskiran, Ibrahim I. (2018). The transcription factor Grainy head primes epithelial enhancers for spatiotemporal activation by displacing nucleosomes. Nature genetics, 50(7), 1011-1020.

[35] Klein, Isaac, Boija, Ann, Afeyan, Lena, Hawken, Susana, Fan, Mengyang, Taatjes, Dylan, Hyman, Anthony. (2021). Partitioning of cancer therapeutics in nuclear condensates: Wolters Kluwer Health.

[36] Klosin, Adam, \& Hyman, Anthony A. (2017). A liquid reservoir for silent chromatin. Nature, 547(7662), 168-169.

[37] Ko, MS, Nakauchi, Hiromitsu, \& Takahashi, Naomi. (1990). the dose dependence of glucocorticoid-inducible gene expression results from changes in the number of transcriptionally active templates. The EMBO journal, 9(9), 2835-2842.

[38] Kraft, Katerina, Magg, Andreas, Heinrich, Verena, Riemenschneider, Christina, Schöpflin, Robert, Markowski, Julia, Andrey, Guillaume. (2019). Serial genomic inversions induce tissuespecific architectural stripes, gene misexpression and congenital malformations. Nature cell biology, 21(3), 305-310.

[39] Kvon, Evgeny Z, Stampfel, Gerald, Yáñez-Cuna, J Omar, Dickson, Barry J, \& Stark, Alexander. (2012). HOT regions function as patterned developmental enhancers and have a distinct cis-regulatory signature. Genes \& development, 26(9), 908-913.

[40] Larson, Adam G, Elnatan, Daniel, Keenen, Madeline M, Trnka, Michael J, Johnston, Jonathan B, Burlingame, Alma L, . . Narlikar, Geeta J. (2017). Liquid droplet formation by HP1 $\alpha$ suggests a role for phase separation in heterochromatin. Nature, 547(7662), 236-240.

[41] Larsson, Anton JM, Johnsson, Per, Hagemann-Jensen, Michael, Hartmanis, Leonard, Faridani, Omid R, Reinius, Björn, . . . Sandberg, Rickard. (2019). Genomic encoding of transcriptional burst kinetics. Nature, 565(7738), 251-254.

[42] Laugsch, Magdalena, Bartusel, Michaela, Rehimi, Rizwan, Alirzayeva, Hafiza, Karaolidou, Agathi, Crispatzu, Giuliano, Kolovos, Petros. (2019). Modeling the pathological long-range regulatory effects of human structural variation with patient-specific hiPSCs. Cell Stem Cell, 24(5), 736-752. e712.

[43] Li, Charles H, Coffey, Eliot L, Dall'Agnese, Alessandra, Hannett, Nancy M, Tang, Xin, Henninger, Jonathan E, Afeyan, Lena K. (2020). MeCP2 links heterochromatin condensates and neurodevelopmental disease. Nature, 586(7829), 440-444.

[44] Li, Jieru, Dong, Ankun, Saydaminova, Kamola, Chang, Hill, Wang, Guanshi, Ochiai, Hiroshi, ... Pertsinidis, Alexandros. (2019). Single-molecule nanoscopy elucidates RNA polymerase II transcription at single genes in live cells. Cell, 178(2), 491-506. e428. 
[45] Li, Jieru, Hsu, Angela, Hua, Yujing, Wang, Guanshi, Cheng, Lingling, Ochiai, Hiroshi, ... Pertsinidis, Alexandros. (2020). Single-gene imaging links genome topology, promoterenhancer communication and transcription control. Nature structural \& molecular biology, 27(11), 1032-1040.

[46] Liang, Hsiao-Lan, Nien, Chung-Yi, Liu, Hsiao-Yun, Metzstein, Mark M, Kirov, Nikolai, \& Rushlow, Christine. (2008). The zinc-finger protein Zelda is a key activator of the early zygotic genome in Drosophila. Nature, 456(7220), 400-403.

[47] Lu, Yi, Wu, Tiantian, Gutman, Orit, Lu, Huasong, Zhou, Qiang, Henis, Yoav I, \& Luo, Kunxin. (2020). Phase separation of TAZ compartmentalizes the transcription machinery to promote gene expression. Nature cell biology, 22(4), 453-464.

[48] Lupiáñez, Darío G, Kraft, Katerina, Heinrich, Verena, Krawitz, Peter, Brancati, Francesco, Klopocki, Eva, Laxova, Renata. (2015). Disruptions of topological chromatin domains cause pathogenic rewiring of gene-enhancer interactions. Cell, 161(5), 1012-1025.

[49] McSwiggen, David T, Mir, Mustafa, Darzacq, Xavier, \& Tjian, Robert. (2019). Evaluating phase separation in live cells: diagnosis, caveats, and functional consequences. Genes \& development, 33(23-24), 1619-1634.

[50] McSwiggen, David Trombley, Hansen, Anders S, Teves, Sheila S, Marie-Nelly, Hervé, Hao, Yvonne, Heckert, Alec Basil, ... Darzacq, Xavier. (2019). Evidence for DNA-mediated nuclear compartmentalization distinct from phase separation. Elife, 8, e47098.

[51] Michael, Alicia K, Grand, Ralph S, Isbel, Luke, Cavadini, Simone, Kozicka, Zuzanna, Kempf, Georg, Pathare, Ganesh R. (2020). Mechanisms of OCT4-SOX2 motif readout on nucleosomes. Science, 368(6498), 1460-1465.

[52] Mir, Mustafa, Stadler, Michael R, Ortiz, Stephan A, Hannon, Colleen E, Harrison, Melissa M, Darzacq, Xavier, \& Eisen, Michael B. (2018). Dynamic multifactor hubs interact transiently with sites of active transcription in Drosophila embryos. Elife, 7, e40497.

[53] Mivelaz, Maxime, Cao, Anne-Marinette, Kubik, Slawomir, Zencir, Sevil, Hovius, Ruud, Boichenko, Iuliia, Fierz, Beat. (2020). Chromatin fiber invasion and nucleosome displacement by the Rap1 transcription factor. Molecular cell, 77(3), 488-500. e489.

[54] Monahan, Kevin, Horta, Adan, \& Lomvardas, Stavros. (2019). LHX2-and LDB1-mediated Tran's interactions regulate olfactory receptor choice. Nature, 565(7740), 448-453.

[55] Nora, Elphège P, Lajoie, Bryan R, Schulz, Edda G, Giorgetti, Luca, Okamoto, Ikuhiro, Servant, Nicolas, Sedat, John. (2012). Spatial partitioning of the regulatory landscape of the Xinactivation centre. Nature, 485(7398), 381-385.

[56] Panigrahi, Anil K, Foulds, Charles E, Lanz, Rainer B, Hamilton, Ross A, Yi, Ping, Lonard, David M, ... O’Malley, Bert W. (2018). SRC-3 coactivator governs dynamic estrogen-induced chromatin looping interactions during transcription. Molecular cell, 70(4), 679-694. e677.

[57] Quintero-Cadena, Porfirio, Lenstra, Tineke L, \& Sternberg, Paul W. (2020). RNA Pol II length and disorder enable cooperative scaling of transcriptional bursting. Molecular Cell, 79(2), $207-$ 220. e208.

[58] Rao, Suhas SP, Huang, Su-Chen, St Hilaire, Brian Glenn, Engreitz, Jesse M, Perez, Elizabeth M, Kieffer-Kwon, Kyong-Rim, Bochkov, Ivan D. (2017). Cohesin loss eliminates all loop domains. Cell, 171(2), 305-320. e324.

[59] Reiter, Franziska, Wienerroither, Sebastian, \& Stark, Alexander. (2017). Combinatorial function of transcription factors and cofactors. Current opinion in genetics \& development, 43, 73-81.

[60] Rodríguez-Carballo, Eddie, Lopez-Delisle, Lucille, Yakushiji-Kaminatsui, Nayuta, UllateAgote, Asier, \& Duboule, Denis. (2019). Impact of genome architecture on the functional activation and repression of Hox regulatory landscapes. BMC biology, 17(1), 1-18.

[61] Rodriguez, Joseph, \& Larson, Daniel R. (2020). Transcription in living cells: Molecular mechanisms of bursting. Annual review of biochemistry, 89, 189-212.

[62] Rodriguez, Joseph, Ren, Gang, Day, Christopher R, Zhao, Keji, Chow, Carson C, \& Larson, Daniel R. (2019). Intrinsic dynamics of a human gene reveal the basis of expression heterogeneity. Cell, 176(1-2), 213-226. e218. 
[63] Sabari, Benjamin R, Dall'Agnese, Alessandra, Boija, Ann, Klein, Isaac A, Coffey, Eliot L, Shrinivas, Krishna, Manteiga, John C. (2018). Coactivator condensation at super-enhancers links phase separation and gene control. Science, 361(6400).

[64] Sexton, Tom, Yaffe, Eitan, Kenigsberg, Ephraim, Bantignies, Frédéric, Leblanc, Benjamin, Hoichman, Michael, Cavalli, Giacomo. (2012). Three-dimensional folding and functional organization principles of the Drosophila genome. Cell, 148(3), 458-472.

[65] Soenmezer, Can, Kleinendorst, Rozemarijn, Imanci, Dilek, Villacorta, Laura, Schuebeler, Dirk, Benes, Vladimir, \& Krebs, Arnaud R. (2020). Single molecule occupancy patterns of transcription factors reveal determinants of cooperative binding in vivo. BioRxiv.

[66] Stavreva, Diana A, Garcia, David A, Fettweis, Gregory, Gudla, Prabhakar R, Zaki, George F, Soni, Vikas, . . Palangat, Murali. (2019). Transcriptional bursting and co-bursting regulation by steroid hormone release pattern and transcription factor mobility. Molecular cell, 75(6), 1161-1177. e1111.

[67] Stergachis, Andrew B, Debo, Brian M, Haugen, Eric, Churchman, L Stirling, \& Stamatoyannopoulos, John A. (2020). Single-molecule regulatory architectures captured by chromatin fiber sequencing. Science, 368(6498), 1449-1454.

[68] Strom, Amy R, Emelyanov, Alexander V, Mir, Mustafa, Fyodorov, Dmitry V, Darzacq, Xavier, $\&$ Karpen, Gary H. (2017). Phase separation drives heterochromatin domain formation. Nature, 547(7662), 241-245.

[69] Su, Jun-Han, Zheng, Pu, Kinrot, Seon S, Bintu, Bogdan, \& Zhuang, Xiaowei. (2020). Genomescale imaging of the 3D organization and transcriptional activity of chromatin. Cell, 182(6), 1641-1659. e1626.

[70] Wang, Liang, Hu, Mingli, Zuo, Mei-Qing, Zhao, Jicheng, Wu, Di, Huang, Li, Bao, Xinhua. (2020). Rett syndrome-causing mutations compromise MeCP2-mediated liquid-liquid phase separation of chromatin. Cell research, 30(5), 393-407.

[71] Williamson, Iain, Kane, Lauren, Devenney, Paul S, Flyamer, Ilya M, Anderson, Eve, Kilanowski, Fiona, Lettice, Laura A. (2019). Developmentally regulated Shh expression is robust to TAD perturbations. Development, 146(19).

[72] Wutz, Gordana, Ladurner, Rene, St Hilaire, Brian Glenn, Stocsits, Roman R, Nagasaka, Kota, Pignard, Benoit, ... Ivanov, Miroslav P. (2020). ESCO1 and CTCF enable formation of long chromatin loops by protecting cohesinSTAG1 from WAPL. Elife, 9, e52091.

[73] Yang, Chuhu, Bolotin, Eugene, Jiang, Tao, Sladek, Frances M, \& Martinez, Ernest. (2007). Prevalence of the initiator over the TATA box in human and yeast genes and identification of DNA motifs enriched in human TATA-less core promoters. Gene, 389(1), 52-65.

[74] Zabidi, Muhammad A, Arnold, Cosmas D, Schernhuber, Katharina, Pagani, Michaela, Rath, Martina, Frank, Olga, \& Stark, Alexander. (2015). Enhancer-core-promoter specificity separates developmental and housekeeping gene regulation. Nature, 518(7540), 556-559.

[75] Zamudio, Alicia V, Dall'Agnese, Alessandra, Henninger, Jonathan E, Manteiga, John C, Afeyan, Lena K, Hannett, Nancy M, Sabari, Benjamin R. (2019). Mediator condensates localize signaling factors to key cell identity genes. Molecular cell, 76(5), 753-766. e756.

[76] Zhu, Fangjie, Farnung, Lucas, Kaasinen, Eevi, Sahu, Biswajyoti, Yin, Yimeng, Wei, Bei, Taipale, Minna. (2018). the interaction landscape between transcription factors and the nucleosome. Nature, 562(7725), 76-81. 